\title{
Hadronic Final States and Their Correlations in pp and Heavy Ion Collisions.
}

\author{
C.A. Ogilvie for the PHENIX Collaboration \\ Iowa State University, Ames, IA, USA
}

\begin{abstract}
One striking observation from experiments at RHIC is the suppression of high-pt particles due to hard-scattered partons losing energy as they propagate through a dense quark-gluon plasma (QGP). The baseline information is single-particle and di-hadron distributions from $\mathrm{p}+\mathrm{p}$ collisions. When measured in $\mathrm{A}+\mathrm{A}$ collisions, these observables provide rich information on the QGP: how the properties of the jet are modified (fragmentation widths and yields), to how the QGP responds to a hard-parton depositing energy along its path.
\end{abstract}

\section{Introduction}

Heavy-ion physics has opened a rich new avenue to explore multibody QCD. By colliding two large nuclei at relativisitic speeds, sufficient energy is deposited into the central region to form a deconfined quark-gluon plasma (QGP). The plasma evolves collectively as a strongly interacting, near-thermalized fluid. These observations have led to a rich set of questions; what is the equation-of-state of a deconfined, multi-parton QCD plasma? and what are the dominant degrees-of-freedom of QGP fluid, e.g. quasi-resonances of interacting partons? ¿From a fundamental perspective the QGP is our first experimental access to the physics of multi-body non-Abelian systems. Also compared to conventional many-body systems interacting via photons, the complications due to self-interacting gluons will expand our knowledge of both general many-body physics as well as non-perturbative QCD.

We have reached a fascinating stage of our experimental endeavors: we are past the initial exploration of forming and identifying the plasma, but we are not yet at the stage where there is a certain, agreed-upon strategy as to how, for example, to determine the plasma's equation of state. The way forward is to increase our control and confidence in diagnostic probes of the plasma.

One promising probe is the passage of hard-scattered partons through the plasma just as it's being formed. As the two nuclei collide the partons within each nucleus have a well-measured, well-known probability to scatter with a high-momentum transfer, e.g. to produce two scattered partons approximately back-to-back each with a $\mathrm{p}_{T}$ above $5 \mathrm{GeV} / \mathrm{c}$. These scattered partons travel through the nascent plasma. Hard-scattered colored partons strongly interact with the deconfined plasma and lose energy via a variety of suggested mechanisms, e.g., bremstrahlung radiation of gluons and elastic collisions with slower partons[1]. It is my goal in this talk[2] to provide an update from the PHENIX collaboration on where we stand in controling and to utilizinbg energy-loss to probe the properties of the plasma.

The PHENIX detector has been in operation since 2000 with two forward/backward muon spectrometer arms to measure quarkonia probes, and two mid-rapidity spectrometer arms to measure photons, $\pi^{0}$, electrons, charged hadrons, quarkonia, and other hadronic resonances. For more details on the PHENIX detector see the recent White paper summary[3]. 


\section{Results}

The spectra of high-pt hadrons from $\mathrm{p}+\mathrm{p}$ collisions as measured by the experiments at RHIC have been compiled by D. d'Enterria[4]. NLO QCD calculations agree with this data very well (Fig. 1) which provides confidence that the baseline of high-pt probes is under control in $\mathrm{p}+\mathrm{p}$ collisions.

The measured high-pt spectra from heavy-ion collisions is significantly reduced compared to that from $\mathrm{p}+\mathrm{p}$ collisions [5], i.e. there are fewer high-pt hadrons in a heavyion collision. One way to plot these data is the ratio of spectra from heavy-ion collisions divided by the spectra from $\mathrm{p}+\mathrm{p}$ collisions (normalized by the larger parton flux in the nuclear events). This ratio $\left(\mathrm{R}_{A A}\right)$ is shown in Fig. 2 for $\pi^{0}, \eta$, and direct photons. The $\mathrm{Au}+\mathrm{Au}$ collisions are nearly head-on, i.e. the $10 \%$ of collisions that have the largest overlap between the incoming nuclei. Both the $\pi^{0}$ and the $\eta$ spectra are suppressed by a factor of 5 compared to proton+proton collisions. In contrast direct-photons below $15 \mathrm{GeV}$ are unsuppressed since they do not interact with the plasma. The hint of sup-

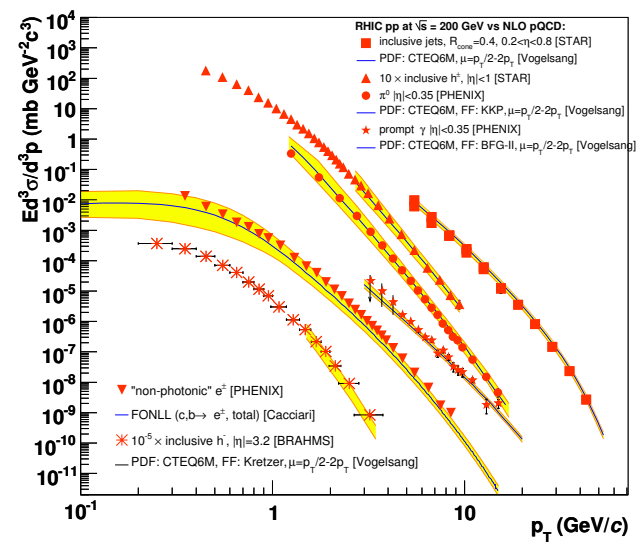

Figure 1: Compiled high-pt spectra from $\mathrm{p}+\mathrm{p}$ collisions at RHIC and their comparison with NLO QCD calculations.[4] pression at higher $p_{T}$ may be due to $\mathrm{p}+\mathrm{n}$ and $\mathrm{n}+\mathrm{n}$ collisions in the nuclear case. The dominant feature that both $\pi^{0}$ and $\eta$ are suppressed by the same amount supports the interpretation that high-pt partons lose energy as travel through the plasma. This is further supported by data from $\mathrm{d}+\mathrm{Au}$ collisions where the hadronic spectra are not suppressed compared with $\mathrm{p}+\mathrm{p}$ indicating that the suppression shown in Fig. 2 is not an initial-state effect.

If the energy lost by a high-momentum parton is radiated by bremstrahlung gluons then we should be able to observe the fate of this radiation. Calculations $[6],[7]$ suggest the radiation is emitted at finite angles relative to the high-pt parton. As a baseline we have measured the yield of associated hadrons that are near in angle to a leading high-pt hadron from $\mathrm{p}+\mathrm{p}$ and $\mathrm{d}+\mathrm{Au}$ collisions and have extracted various jet-widths and fragmentation properties [8] [9]. We then compare this baseline to jet-structures in $\mathrm{A}+\mathrm{A}$ collisions. As an example Fig. 3 shows the transverse momentum $p_{\text {out }}$ of associated hadrons with respect to the axis defined by a highpt $\pi^{0}$ for both $\mathrm{p}+\mathrm{p}$ collisions and central $\mathrm{Cu}+\mathrm{Cu}$ collisions. The $p_{\text {out }}$ distibution is

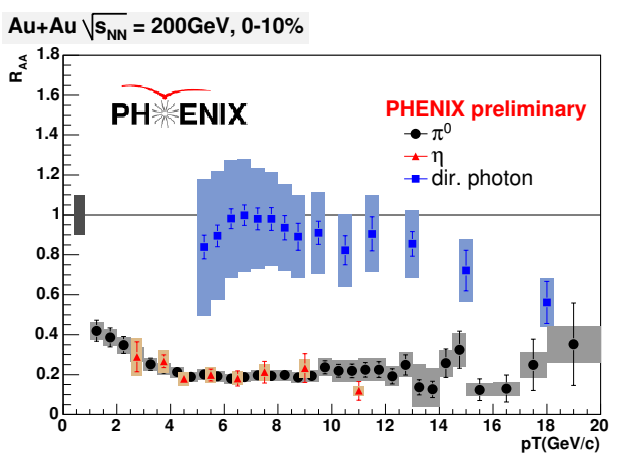

Figure 2: Ratio of the measured spectra of $\pi^{0}, \eta$, and direct photons from central $\mathrm{Au}+\mathrm{Au}$ collisions divided by the spectra from $\mathrm{p}+\mathrm{p}$ collisions. 
broader in the heavy-ion data suggesting that the gluon radiation from the energyloss eventually fragments and increases the yield of associated hadrons at finite angles.

The suppression of high-pt hadrons and the increased yield of associated hadrons at finite angles are two pieces of evidence that high-pt partons lose energy as they travel through the plasma. A complementary question is how does the plasma respond to the impact of a high-pt parton. To explore this we have measured the azimuthal distribution $\Delta \phi$ of associated hadrons with respect to angle defined by a leading highpt hadron. An example is shown in Fig. 4 which contains the angular distribution of hadrons with $1<p_{T}<2.5 \mathrm{GeV} / \mathrm{c}$ with respect to a trigger hadron between $2.5<$

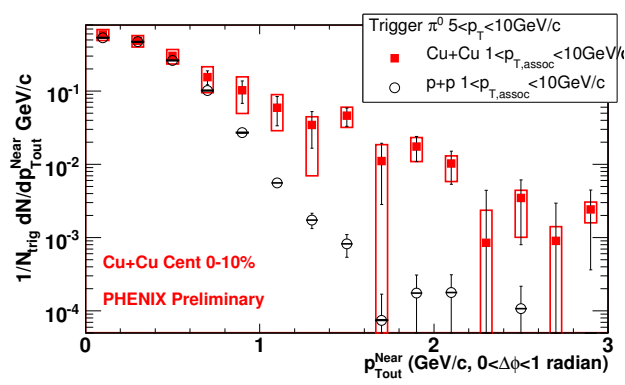

Figure 3: The $p_{\text {out }}$ distributions from $\mathrm{p}+\mathrm{p}$ and central $\mathrm{Cu}+\mathrm{Cu}$ collisions.

$p_{T}<4 \mathrm{GeV} / \mathrm{c}[10]$ from central $\mathrm{Au}+\mathrm{Au}$ collisions. The data shown as squares (black) are the original correlation while the data in circles (red) are after correcting for the underliying event which has an overall modulation due to the known elliptic flow. The near-side peak at $\Delta \phi \sim 0$ corresponds to hadrons from the fragmentation of the same high-pt parton. In $\mathrm{p}+\mathrm{p}$ collisions the far-side peak is at $\Delta \phi \sim \pi$ which corresponds to the fragmentation of two back-to-back high-partons[8]. In striking contrast the central $\mathrm{Au}+\mathrm{Au}$ data have a side peak approximately 1 radian away from the expected location of the far-side peak.

This side-peak has been characterized in several ways: perhaps the most transparent is fitting with a gaussian displaced from $\pi$ by $\mathrm{D}$ radians. The fitted parameter $\mathrm{D}$ for several centralities from both $\mathrm{Au}+\mathrm{Au}$ and $\mathrm{Cu}+\mathrm{Cu}$ collisions[10] smoothly increases with centrality with the results falling on a common curve from different colliding systems. This is consisent with the peak being a property of the matter that is formed during the collision, i.e. a response of the matter due to the passage of the jet. It is not yet known what this response is, though there have been predictions of a mach-cone being formed in the fluid surrounding the passage of a supersonic high-pt parton[11].

\section{$3 \quad$ Next Steps}

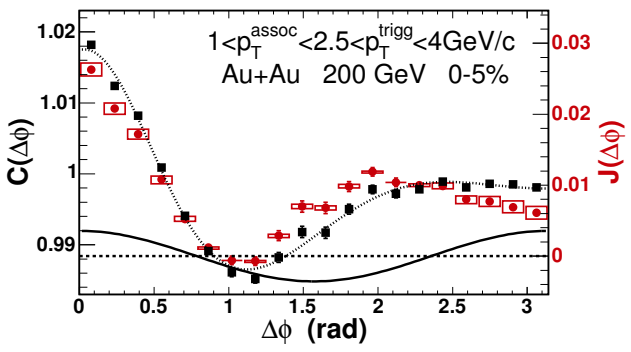

Figure 4: The azimuthal distribution of associated hadrons with respect to the axis defined by a leading trigger hadron for central $\mathrm{Au}+\mathrm{Au}$ collisions. The data shown as squares (black) are the original correlation while the data in circles (red) are after correcting for the underliying event which has an overall modulation due to the known elliptic flow.

Recapping the main message of this talk: we have formed a strongly interacting QGP in experiments at RHIC and are learning how to use high-pt probes to quantify the properties of this matter. Partons traveling through this medium lose energy leading to a reduced yield 
of high-pt hadrons. At the same stage an increase in the number of hadrons correlated with the leading hadron is consistent with the fragmentation of radiated, bremstrahlung gluons.

At least two key measurements are needed to increase our control and understanding of these probes. Charm and beauty quarks are predicted to lose less energy as they travel through the plasma. Calculations predict less gluon radiation due to larger gluon interference during the heavy-parton's multiple-scattering, i.e. a medium dead-cone effect. Measurements of the sum of semi-leptonic decays of charm and beauty[12] suprisingly show a high-pt suppression similar to that for pions. This is a challenge for models: if they can reproduce both light- and heavy-quark energy-loss, this will increase the confidence in how gluon radiation is modeled and increase our ability to use these probes of the QGP. Experimentally we also need to separate the spectra of charm from beauty: this required the funded upgrade of a high-precions silicon vertex detector that will be installed during 2009/10.

The second next step is to control the path-length of high-pt probes. The amount of energy-loss depends on both the density of the medium and the path-length that is traveled by the parton. How to best disentangle these two effects is an open question: work proceeds on multiple fronts; studying energy-loss versus reaction plane, centrality, and for different size colliding systems. Part of the difficulty is the strong energy-loss means that the set of partons that survive to produce high-pt hadrons, come from hard-collisions that occur predominantly near the surface of the dense matter. One possible method to alter this surface-bias is to select events that have two back-to-back high-pt hadrons. On average these partons will have traveled through similar lengths of dense matter and lost similar amounts of energy. This will shift the surface bias more towards the center of this collision.

\section{Acknowledgments}

The author acknowledges funding from the DOE.

\section{References}

[1] Majumder, A. nucl-th/0702066

[2] Slides:

http://indico. cern. ch/contributionDisplay. py? contribId=231\&sessionId=6\&conf $I d=9499$

[3] Adcox, K. et al., Nucl. Phys. A757 184 (2005).

[4] d'Enterria, David, J. Phys. G34 S53-S82 (2007).

[5] Adcox, K. et al., Phys. Rev. Lett. 88022301 (2002).

[6] Majumder, A. and Wang, Xin-Nian, Phys. Rev. D72 034007 (2005).

[7] I. Vitev, Phys. Lett. 63078 (2005).

[8] Adler, S.S. et al. Phys. Rev. D74 072002 (2006).

[9] Adler, S.S. et al. Phys. Rev. C73 054903 (2006).

[10] Adler, S.S. et al. Phys. Rev. Lett. 97052301 (2006).

[11] J. Casalderrey-Solana, E.V. Shuryak, and D. Teaney, J. Phys. Conf. Ser. 2722 (2005).

[12] Adare, A. et al. Phys. Rev. Lett. 98172301 (2007). 